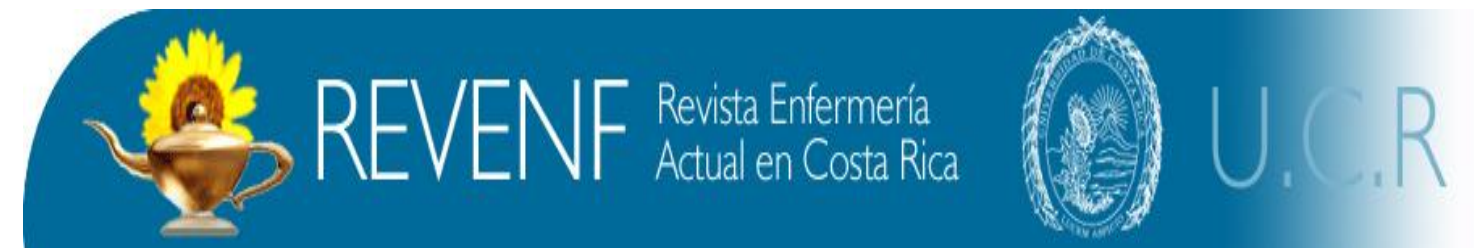 \\ Revista Semestral Number 12-Abril - Setiembre 2007 ISSN 1409-4568 \\ Como citar este artículo
}

Matamoros Quesada, Karla y otros. Percepción de los usuarios y usuarias sobre el quehacer de enfermería en el primer nivel de atención. Rev. Enfermería Actual en Costa Rica [en línea]. Abril-Setiembre 2007 No.12 [citado (fecha)]. Disponible World Wide Web: < http://www.revenf.ucr.ac.cr/percepcion.pdf> ISSN 1409-4568

\section{PERCEPCIÓN DE LOS USUARIOS Y USUARIAS SOBRE EL QUEHACER DE ENFERMERÍA EN EL PRIMER NIVEL DE ATENCIÓN ${ }^{1}$}

\author{
Matamoros Quesada Karla ${ }^{2}$ \\ Natalia Obando Godínez ${ }^{2}$ \\ Susana Rivera Volio ${ }^{2}$ \\ Dahianna Romero Guillén ${ }^{2}$ \\ Christian Torres Acuña ${ }^{3}$ \\ Ana Guzmán Aguilar ${ }^{4}$
}

\section{Resumen}

Este artículo describe los resultados de un estudio sobre la percepción que tienen los usuarios(as) adultos acerca del quehacer de Enfermería en el Primer Nivel de Atención. Se realizó en cinco EBAIS seleccionados del área de salud de Cartago, con un enfoque cuantitativo no experimental que permitió conocer las causas de este fenómeno social. Además se planteó un diseño transaccional correlacional. Se utilizó la teoría de Imogene King para el análisis de los resultado obtenidos, la percepción es un elemento del sistema personal fundamental para lograr las transacciones necesarias y el logro de objetivos en la intervención de enfermería.

La información obtenida permitió identificar una percepción errónea de las personas adultas acerca del rol de Enfermería en el primer nivel de atención, debido principalmente a varios factores, entre ellos: ausencia de profesionales de Enfermería en este nivel, el desconocimiento del perfil y funciones de cada uno de los miembros del equipo de Enfermería y el traslape de funciones entre los miembros del personal; lo cual no permite identificar de manera clara las funciones de cada uno de ellos. La mayoría de las personas adultas han tenido algún acercamiento con el personal de enfermería y poseen opiniones favorables; sin embargo, la experiencia está revestida de misticidad y vocación. Por otra parte, la visión biomédica de la salud es evidente en las expectativas de los usuarios (as) que continúan viendo a Enfermería como una profesión dependiente de la medicina. El análisis inferencial permitió establecer una asociación significativa entre la calificación del cuidado y la educación que se brindó.

En general, se encontró respuestas tanto positivas como negativas en torno a percepción de la enfermería basadas en la experiencias previas y el contacto con las personas y no con el conocimiento objetivo acerca de la profesión.

Palabras claves: percepción, Enfermería, cuidado, primer nivel de atención.

\footnotetext{
${ }^{1}$ Fecha de recepción: Diciembre 2006

Fecha de aceptación: Marzo 2007

${ }^{2}$ Licenciadas en Enfermería. Escuela de Enfermería, Universidad de Costa Rica.

${ }^{3}$ Licenciado en Enfermería. Escuela de Enfermería, Universidad de Costa Rica.

${ }^{4}$ Profesora de la Escuela de Enfermería de la Universidad de Costa Rica. Magistra en Planificación

Curricular. E mail: $\underline{\text { anag@gmail.com }}$
} 


\begin{abstract}
This article describes the results of a study on perception that has the adult people about the task of nursing in the first level of attention. This study was realized in five selected EBAIS of Cartago area. The methodology applied was a non-experimental quantitative approach and a correlation transactional design that permits to know the causes of this social phenomenon. It was used the theory of Imogene King for the obtained analysis of the result. The perception is an element of the personal system fundamental to obtain the necessary transactions and the profit of objectives in intervention of nurse. The obtained data allowed to mainly identify an erroneous perception of the adult people about the roll of Nursing in the first level of attention, which had to several factors, among them: absence of professionals of Nursing in this level, the ignorance of the profile and functions of each one of the members of the equipment of Nursing and overlaps of functions between the members of the personnel; which does not allow to identify of clear way the functions of each one of them. Most of the adult people they have had some approach with the Nursing personnel and have favorable opinions; nevertheless, the experience is had with mystic and vocation. On the other hand, the biomedical vision of the health is evident in the expectations of the people who continue seeing Nursing like a dependent profession of the medicine. The inferential analysis allowed establishing a significant association between the qualification of the care and the education that offered. In general, one as much was positive answers as negative around perception of the Nursing based on the previous experiences and the contact with the people and not with the objective knowledge about the profession.
\end{abstract}

Key words: perception, Nursing, care, first level of attention. 


\section{Introducción}

El presente artículo evidencia la problemática relacionada básicamente con la percepción que poseen las personas con respecto al quehacer de Enfermería en el Primer Nivel de Atención. Se aborda desde los postulados teóricos de Imogene King, por ser esta autora quien esboza diferentes concepciones filosóficas relacionadas con la profesión y el quehacer de Enfermería y dentro de estas, la percepción, la cual da fundamento al estudio.

En la revisión bibliográfica realizada se encontró que en España, Miriam y Sonia Albert Sobrino, en su estudio titulado: "¿Enfermería a examen: cómo nos ve la sociedad?" (2002), se evidenció que casi la mitad de los entrevistados (as), identifica como función de las enfermeras (os) cuidar personas enfermas, mientras que el porcentaje restante atribuyen funciones técnicas o de ayuda al médico (a).

El Colegio Oficial de Enfermería de Málaga (2005) llevó a cabo una investigación donde se indaga la percepción de los profesionales sobre el desarrollo de la Enfermería en dicha provincia. La conclusión más relevante de este estudio fue que la población no puede apreciar a cabalidad la intervención de Enfermería ya que ésta queda opacada y desvanecida por la actuación médica.

En México, González C, Delgado B y Ayala S (2001) realizaron un estudio sobre la percepción que tienen las personas de la Enfermería en Monterrey, Nuevo León encontrándose que más de la mitad $(52 \%)$ de las personas encuestadas consideran a Enfermería como personal de salud en general. Además, más de la mitad de éstas distingue al personal de
Enfermería por su uniforme y no por sus acciones. Por otra parte, M.E. María Guadalupe Moreno Monsívais y otros (2002), en la ciudad de Monterrey, encontraron que las características del cuidado de la enfermera (o) se describen como: "conforta", "explica y facilita" y "mantiene relación de confianza".

En Chile, un artículo realizado por J. Yañez y J. Araya (1999), titulado "La perspectiva de la Enfermería profesional" menciona que tradicionalmente, a la enfermera se le han atribuido valores como la caridad, altruismo, abnegación, vocación y sumisión, frente a otros profesionales, disminuyendo la autonomía de sus decisiones.

En Bogotá, Rosita Daza de Caballero y otras (2005) en su investigación “Análisis crítico del cuidado de Enfermería: interacción, participación y afecto", establecen diferencias entre el desempeño de la enfermera (o) y el del auxiliar de Enfermería. Refieren que: "gracias a la toca $\mathrm{y}$ a la cinta que hacen parte del uniforme de las jefes, es que las diferenciamos, además por el grado de responsabilidad frente al cuidado".

En Puerto Rico, G. Pizarro y otros realizaron un estudio llamado "Estigma social sobre Enfermería", donde se concluyó que la población de Puerto Rico afirma que Enfermería es una profesión que requiere mucho esfuerzo $y$ es considerada como indispensable por la sociedad. Además citan factores que inciden en la adquisición de un estigma negativo para la misma, como por ejemplo programas y comedias de televisión donde se degrada la imagen de estos profesionales, y en una gran minoría la experiencia propia. Los investigadores afirman que para la sociedad de Puerto 
Rico la profesión de Enfermería es digna de admirar, respetar, honrar y valorizar por el trabajo arduo en beneficio de la sociedad.

En Cuba, Hernández Vergel, Rodríguez Brito, Jiménez Pérez y Rangel Marín (2002), elaboraron una investigación titulada "Imagen Social de la Enfermería". Se pudo observar que en la población estudiada el 93,55\% respondió que el personal de Enfermería puede ser de uno u otro sexo. Ello corrobora que las costumbres actuales evolucionan hacia el acercamiento de los papeles masculinos y femeninos. Además se encontró que, según la población, las actividades fundamentales de Enfermería son inyectar y curar, ayudar a los enfermos interesándose por sus problemas y ayudar al médico, entre otros resultados.

Estos estudios demostraron la diversidad de opiniones con respecto a Enfermería. Se han observado tanto posiciones favorables, especialmente en las investigaciones realizadas en el Caribe, como desfavorables, donde enfermeros y enfermeras se han visto inmersos en la complejidad del fenómeno del estereotipo, el prejuicio y la divagación del concepto, rol y quehacer de Enfermería.

Por lo tanto, fue interesante e importante indagar la temática en nuestro país. En Costa Rica hay ausencia de investigaciones relacionadas con esta premisa, por lo que el abordaje de la misma contribuye al desarrollo de la Enfermería ya que se ha notado que las investigaciones citadas anteriormente crean conocimiento para la profesión y resaltan la importancia de la enfermera (o) en los diferentes escenarios.
Por otro lado, contribuye de manera positiva en el avance del conocimiento científico, con miras a trazar nuevas metas que permitan replantear el ejercicio profesional en el Primer Nivel de Atención. Además, pretendió mostrar la necesidad de la presencia del profesional de Enfermería en este nivel evidenciada por dos argumentos principales: primero, la invisibilización del profesional dentro de las comunidades y los EBAIS y el traslape de funciones que crea la presencia de personal técnico y auxiliar en estos establecimientos, creando confusión en los usuarios(as) con respecto al perfil de cada uno de los miembros del equipo de Enfermería. Segundo, la preparación académica de los y las enfermeras hacen de este profesional, el personal idóneo para cumplir los objetivos planteados al crear los Equipos Básicos de Atención Integral en Salud (EBAIS), que son en términos generales, brindar atención integral al niño (a), adolescente, mujer embarazada y adulto mayor, por lo que las comunidades no están recibiendo este valioso recurso en pro de la mejora en la calidad de los servicios de salud.

El objetivo general de este estudio fue analizar la percepción de las personas adultas usuarias de los servicios de salud de 5 Equipos Básicos de Atención Integral en Salud (EBAIS) del Área Central de Cartago, sobre el rol que desempeña el personal de Enfermería en el Primer Nivel de Atención.

\section{Materiales y métodos}

Esta investigación se desarrolló en cinco etapas estructurales: I Etapa: Diagnóstico Situacional, II Etapa: Elaboración de la propuesta de investigación, III Etapa: 
Elaboración y aplicación de instrumentos, IV Etapa: Procesamiento de datos y análisis de resultados y $\mathrm{V}$ Etapa: Elaboración del informe.

Se diseñó un enfoque cuantitativo no experimental, de tipo exploratorio en primera instancia debido a que es un tema poco estudiado en el país, posteriormente se realizó una descripción de los hallazgos $\mathrm{y}$ finalmente, se relacionaron algunas variables de interés para determinar si existía relación entre ellas. Los datos fueron recolectados en un momento único por lo que se caracteriza como transeccional.

\section{Población y muestra}

La población total del área estudiada estaba compuesta por 104.863 usuarios(as) distribuidos en 21 EBAIS. A partir de esta población, se tomó una muestra estadísticamente representativa. Se seleccionó una muestra por conglomerados, la cual correspondió a los EBAIS Manuel de Jesús Jiménez 1, El Carmen, La Lima, Caballo Blanco, y Oriente 2. Este tipo de muestreo es "...un procedimiento aleatorio de un conjunto de individuos, que busca seccionar la población total en grupos, como fase previa a la extracción muestral." (Rojas, L. 2004).

A partir de la muestra por conglomerados, se extrajo una segunda muestra, ello mediante la utilización del muestreo polietápico posterior, el cual pretende reconocer como unidad muestral una subdivisión de los conglomerados elegidos, al escoger aleatoriamente sólo una parte de los integrantes del conglomerado (Rojas, L. 2004: p.176).

\section{Marco muestral}

Se llevó a cabo una selección aleatoria de conglomerados con la que se obtuvo una muestra final de 240 usuarios y usuarias, en 5 EBAIS, elegidos al azar.

Se determinó además el error máximo correspondiente al estudio, el cual correspondió a $5.3 \%$ para un nivel de confianza del $90 \%$

\section{Instrumentos y técnicas}

Se elaboró una entrevista semiestructurada a informantes clave (líderes comunales, personal de Enfermería que labora en los EBAIS seleccionados, jefa del departamento de Enfermería del área) y un cuestionario auto administrado, previamente validado, que se aplicó a la muestra seleccionada de usuarios(as)

El cuestionario se estructuró en dos partes: en la primera, se elaboraron preguntas generales como edad, género, nivel educativo y estado civil, con el objetivo de ubicar socioeconómicamente a la población. En la segunda parte, se incluyeron preguntas que responden directamente a las dimensiones y variables planteadas dentro del sistema de variables. Para este instrumento se contó con preguntas abiertas, cerradas y mixtas.

Una vez concluida la etapa de recolección de los datos, se utilizó el programa informático Excel, para el estudio de los mismos.

Como parte del análisis estadístico, se elaboraron estadísticas bi-variadas, para la cual se usaron tablas de contingencia y 
Chi Cuadrado para establecer relaciones de dependencia entre los elementos.

Falta ética

\section{Resultados}

Entre los resultados más relevantes se destacan los que se describen a continuación.

\section{Funciones de Enfermería en el Primer Nivel de Atención}

Se encontró que de las 218 personas entrevistadas, la mayoría estuvo de acuerdo en que la principal función de Enfermería es tomar la presión arterial, pulso, temperatura y respiración, seguido de brindar primeros auxilios en caso de emergencia, aspecto con un número de respuestas positivas de 205 . El ayudar a las personas enfermas, preocupándose por sus dificultades obtuvo un número de respuestas positivas de 195 .

Se observó que, en general, los y las participantes opinaron que las funciones están relacionadas con trabajos técnicos y de ayuda al prójimo.

\section{Atención de la persona adulta mayor en el hogar}

Para la pregunta ¿Conoce usted si alguna de estas personas (Enfermera, Auxiliar, Asistente Técnico en Atención Primaria otra persona) le brinda atención a la persona adulta mayor en el hogar?, el $52.9 \%$ dice no conocer y un $47.1 \%$ refiere sí conocer este servicio. Sin embargo, para los EBAIS Oriente 2 y Manuel de Jesús Jiménez 1 , un $54 \%$ y un $52 \%$ respectivamente dijeron sí conocer acerca de la atención al adulto mayor, hecho que no es constante en los demás Centros de Salud, pues en ellos la mayoría de las personas dicen no conocer acerca de esta atención.

Al preguntar a los usuarios(as) que afirmaron conocer acerca de la atención a la persona adulta mayor sobre quién es la persona que ofrece esta atención, se observó que el $43.75 \%$ de las personas manifiestan que es la enfermera quien la brinda, con excepción del EBAIS La Lima en el que el $40.9 \%$ dijo que era la encargada de brindar la atención primaria (ATAP). Así mismo en el total de los EBAIS, el $24.12 \%$ refirió que era la ATAP y $13.39 \%$ dijo que es la auxiliar de Enfermería.

\section{Conocimiento acerca de la existencia de la Enfermera profesional}

De la muestra global, el $58.33 \%$ de los encuestados y encuestadas dijo no conocer a la Enfermera Profesional y un $41.67 \%$ dijo sí conocerla.

Sin embargo, se observaron algunas particularidades o discrepancias, por ejemplo en la comunidad de Manuel de Jesús Jiménez el $67 \%$ dice sí conocerla. En los demás EBAIS la mayoría no conocen a la enfermera profesional.

Se observó que el $59 \%$ de los y las participantes identifican al auxiliar de Enfermería como si fuese el o la profesional.

Educación brindada por el personal de Enfermería a usuarios(as) de los EBAIS en estudio 
En este apartado, el $38.75 \%$ refirió no haber recibido ningún tipo de información por parte del personal de Enfermería, mientras que la mayoría, es decir, el $61.25 \%$ de la población total sí recibió educación en algún momento.

En cinco de los cuatro EBAIS, las personas refieren sí haber recibido información por parte del personal de Enfermería, excepto en el EBAIS de El Carmen 1 en el cual la mayor cantidad de personas mencionaron no haberla recibido $(62.5 \%$ de la población en este centro de salud). El EBAIS con mayor porcentaje de personas que sí recibieron educación correspondió a Oriente 2 con $75 \%$.

Grado de satisfacción de usuarios (as) con respecto a la atención ofrecida por el personal de Enfermería.

Del $61.25 \%$ de personas que sí recibieron información, el 30.6\% se encuentran muy satisfechas, y el $55.78 \%$ se siente satisfecho.

\section{Actitud tomada al recibir la información} por parte del personal de Enfermería

La credibilidad de la información brindada por el personal de Enfermería en los cinco EBAIS es muy alta, ya que el $75.51 \%$ de las personas que han recibido información la acepta y cree en ellas. Solamente una minoría $(15.64 \%)$ duda y busca corroborarla con el médico u otro personal de salud. Es muy importante destacar que ninguna persona, refirió no creer al personal de Enfermería.

Campos de trabajo en los que consideran que puede desempeñarse una enfermera
Se observa que las principales opciones marcadas por los usuarios(as) entrevistados son los Hospitales, los EBAIS y las Clínicas con valores que oscilan entre el $80 \%$ a $85 \%$ de la población, esto demuestra que todavía se mantiene una predisposición a ubicar al personal de Enfermería en los contextos más tradicionales donde se atiende en su mayoría procesos mórbidos de salud y en los cuales generalmente las personas tienen mayor acceso y contacto con Enfermería. Por otra parte, las opciones con menor frecuencia de repuesta son las empresas, fábricas, los centros educativos y la comunidad con valores que varían de $59 \%$ a $67 \%$.

\section{Preferencia de género del personal de Enfermería que atiende en los EBAIS}

En todos los EBAIS estudiados, las personas refirieron no tener preferencia de género del Personal de Enfermería a la hora de la atención, ya que 152 personas no tienen preferencia para ser atendidos por enfermeras o enfermeros; 151 usuarios(as) refieren el mismo criterio con respecto al auxiliar de Enfermería y 155 individuos no tiene predilección para ser atendido por un o una ATAP. Sin embargo, una porción nada despreciable de encuestados (as) de todos los EBAIS prefieren ser atendidos por un miembro del personal de Enfermería de sexo femenino; 80 personas prefieren ser atendidos por enfermeras, 78 por auxiliares de Enfermería de sexo femenino y 75 por mujeres ATAP`s.

Solamente en el EBAIS de Caballo Blanco y en Manuel de Jesús Jiménez prefiere la atención de un varón, 3 personas prefieren ser atendidos por 
enfermeros, 4 por auxiliares de Enfermería de sexo masculino y 3 por varones ATAP`s.

Grado de satisfacción de usuarios(as) de los EBAIS en estudio con respecto a los cuidados brindados por el personal de Enfermería durante la consulta

En este apartado se observa que las respuestas oscilan entre excelente y bueno, pues se obtuvo $42.5 \%$ para la primera y $42.9 \%$ para la segunda. Un $9.2 \%$ califica el cuidado como regular y un porcentaje pequeño de usuarios(as) lo califica como deficiente $(0.8 \%)$. Es importante destacar que ninguna persona calificó el cuidado como malo

Forma en que identifican los (as) usuarios(as) a una enfermera o enfermero

En este aspecto se obtuvo que el $62.1 \%$ de personas reconocen a la enfermera como la persona que toma la presión, mide y pesa a los usuarios(as); el $48.75 \%$ la o lo reconoce por el uniforme que viste $\mathrm{y}$ el $18.75 \%$ porque es quien coordina actividades en el EBAIS. Solamente un $15 \%$ la reconoce porque es la persona que da charlas sobre temas de salud.

\section{Opinión sobre la Enfermería en general}

La mayoría de personas posee opiniones muy favorables con respecto a la Enfermería en general. Hacen referencia a que es una profesión muy importante y útil para la sociedad. En todos los EBAIS se encontraron comentarios positivos como: "es excelente", "son amables" y "brindan un servicio muy importante".

Sin embargo, también se evidencia que se sigue considerando a la Enfermería como una disciplina altamente dependiente de los médicos. Muchos de los encuestados hicieron comentarios como: "son un complemento del médico", "orienta cuando el médico da recomendaciones".

Por otro lado, muchas respuestas estuvieron orientadas a destacar la caridad y vocación de la Enfermería como disciplina. Comentarios como los siguientes lo evidencian: "es un don de Dios amar y servir al prójimo", "es un área de mucha vocación, conocimiento y relaciones humanas".

Muy pocas personas expresaron comentarios negativos sobre la Enfermería. Sin embargo, los que sí lo hicieron orientaron sus respuestas a las características personales del enfermero(a), como por ejemplo que son ineficientes.

\section{Importancia social de la Enfermería}

En una escala de 1 al 10 de acuerdo a la importancia social (donde 10 es el mayor valor otorgado), en todos los EBAIS el médico obtuvo la mayor frecuencia de respuestas con valor de 10. Además, en todos los EBAIS la enfermera ocupó el segundo lugar, excepto en Manuel de Jesús Jiménez, donde fue superada por el nutricionista.

\section{Opinión acerca de si un hijo o hija deseara estudiar Enfermería}

Se observa que el $83.75 \%$ de la población dice que si les gustaría que un hijo o hija estudiase Enfermería, el $9.58 \%$ dijo que no le gustaría y un $6.67 \%$ no dio opinión al respecto. Los motivos por los que sí les gustaría que sus hijos (as) estudiaran enfermería son muy variados. En orden 
de frecuencia se obtuvieron los siguientes resultados: es una profesión muy importante para la sociedad; para que de esta forma ayuden a las demás personas y a la familia cuando haya algún enfermo y es muy humanitaria.

Dentro de los motivos mencionados por la población por los que no les gustaría que un hijo o hija estudiara Enfermería, se encuentran: es una profesión de mucho sacrificio, es muy complicada; no es rentable y puede conllevar a alto riesgo de contagio.

\section{Discusión}

La percepción que los usuarios(as) tienen acerca del rol que desempeña el personal de enfermería en el Primer Nivel de Atención, es una forma de conocer cómo interpretan y principalmente que expectativas tienen ellos y ellas del trabajo de enfermería, y relacionarlas primordialmente con carencias y fortalezas en la profesión para lograr un mejor desempeño.

Se destacó entre los resultados la ausencia de un profesional de Enfermería en el Primer Nivel que brinde atención directa al usuario(a), por lo que la labor del profesional es desconocida para estos, ya que al ser el auxiliar de enfermería el principal encargado de brindar la atención, es a este personal a quien los usuarios $y$ usuarias reconocen principalmente como enfermera(o) y sus labores, que son técnicas (pesar, tomar la presión, entre otras), son parte de enfermería, pero no únicas.
Por otro lado existe un traslape de roles entre las (los) auxiliares de Enfermería y las enfermeras (os) en el Primer Nivel de Atención, lo que provoca confusión en los usuarios(as) y no tienen claridad de quién es la enfermera o enfermero, auxiliar o técnico de Atención Primaria y se refieren indistintamente a una (o) u otra (o). Esta confusión se agrava por el hecho de que el uniforme es la segunda manera más común de identificar a la enfermera (o), lo cual da pie al error, ya que muchas personas ajenas a Enfermería utilizan uniformes semejantes.

Lo anteriormente citado es semejante a los datos obtenidos por Sobrino y Sobrino (2002), donde determinaron que las personas que participaron en su investigación identificaron a las enfermeras con cualidades técnicas, vocacionales y atuendos clásicos como cofia, vestido blanco, capas, etc.

Asimismo se puede anotar que el uniforme también es una importante identificación para enfermería en varias partes del mundo, González, Delgado y Araya en el año 2000, en un estudio realizado en México sobre la percepción que tienen las personas de la enfermería más de la mitad refieren que distinguen al personal por su uniforme $\mathrm{y}$ no por sus acciones.

Es aquí donde se pierde mucho del verdadero ser de Enfermería, encargada de brindar educación útil y oportuna a los usuarios(as), en pro de mejorar la salud de los mismos. Para Bertilia y Josefa Retana (1984) uno de los roles de enfermería en salud comunitaria es el rol de educadora y se afirma en todos los niveles de atención . Enfermería tiene la 
responsabilidad de promover, organizar y ejecutar actividades educativas para los usuarios (as) y la comunidad

Como consecuencia de la ausencia de un profesional de Enfermería en la atención directa, la principal representación de Enfermería a los usuarios(as) es por el personal auxiliar. Ellos desde su área desempeñan una buena labor, pero no pueden sobrecargarse de trabajo, por lo que las labores educativas son las que quedan de lado, lo cual no es congruente con el accionar del Primer Nivel de Atención, ya que a este nivel lo ideal es favorecer la educación en salud para evitar problemas que pueden prevenirse si se educa de manera eficiente a los usuarios (as).

Existe un desconocimiento general sobre la profesión y una invisibilización de la enfermera dentro del primer nivel de atención Cano Caballero(2004) realizó un análisis a la luz de la perspectiva de género $y$ menciona que la indefinición de las funciones propias de enfermería ha hecho que asuma actividades dispersas que pueden ser desarrolladas por personal poco calificado y bien entrenado. Estos datos coinciden con los encontrados en otros estudios como el de Miriam y Sonia Albert Sobrino (2002), donde se identifica que la función del profesional de Enfermería es cuidar enfermos $y$ de igual manera $J$ Yanes y J. Araya (1999) realizan un estudio en donde se dice que tradicionalmente a este y esta profesional se le han atribuido valores como la caridad, altruismo, abnegación vocación y sumisión, frente a otros profesionales, disminuyendo la autonomía de sus decisiones. Las respuestas están encaminadas dentro de la problemática en estudio y evidencian la percepción idealizada y mítica acerca de la profesión, las cuales no son desfavorables pero pueden limitar la labor que las enfermeras y enfermeros pueden realizar.

Llama la atención que el estatus otorgado a la Enfermería es alto, pues ocupó el segundo lugar en la escala de importancia social. Sin embargo, medicina ocupó el primer lugar ya que tradicionalmente ha sido una de las profesiones que ha mantenido un alto estatus social a través de la historia; sin embargo, la Enfermería a pesar de ser una profesión marcada por el género, y que es vista como de complemento al médico, debe cuidar, mantener y superar las expectativas que tiene la población sobre la disciplina; pues tal como lo confirma la investigación del Colegio oficial de Enfermería en Málaga, donde se encontró que el rol del profesional de Enfermería queda difuminado en el acto médico y en la brevedad del proceso. Además, Sobrino y Sobrino ( 2002) encontraron que un $64 \%$ de la población entrevistada asegura que dichas funciones se desarrollan bajo las ordenes de un médico .

Con respecto al análisis inferencial, se encontró que existe asociación significativa entre la calificación del cuidado y si se brindó educación a los usuarios(as), por lo que brindar educación influye en la apreciación del cuidado. Además, se pudo observar que entre mejor es la educación y cuidado ofrecido por el personal, se obtendrá mayor calificación de la labor de Enfermería. 
Es importante resaltar que durante el tiempo de aplicación de los cuestionarios el personal presente en los EBAIS fue femenino. $\mathrm{Al}$ respecto se puede señalar que con el rol femenino se identifica la gentileza, la piedad, la compasión, la prudencia y la belleza. (Barton:1971 p.101).

Así mismo según lo expuesto por Virginia Enciso Huerta (1997) en su artículo "Genero y trabajo: La Enfermería" refiere que a la mujer corresponden rasgos que vinculan la feminidad a la sumisión, la compasión, la pasividad y la emotividad compatibles con las funciones de la enfermera (o).

Uno de los alcances más significativos fue que, mediante un trabajo científicamente se obtuvo un acercamiento a la realidad sobre la percepción de enfermería en el primer nivel de atención. Estos resultados son similares a los obtenidos en el estudio realizado por González C. Delgado B y Ayala S (2000) donde se concluye que el quehacer de Enfermería se encasilla dentro d e la práctica hospitalaria o bien dentro de la práctica de la curación desligándola d e la promoción de la salud y la prevención de las enfermedades, lo cual corresponde al paradigma de la atención en Costa Rica.

Dentro las limitaciones que más afectaron el desarrollo de la investigación fue el desconocimiento de los usuarios(as) acerca de quienes conforman el personal de Enfermería y que actividades desempeñan cada uno, lo que dificultó el posterior análisis de los resultados.

\section{Conclusión}

$\checkmark$ La población ignora cada uno de los roles del personal de Enfermería dentro del Primer Nivel de Atención, dejando en evidencia que los antiguos, pero bien sabidos atributos mítico - religiosos permanecen fuertemente arraigados en las personas y que la percepción hacia Enfermería gira entorno a ellos, sin que se desliguen de la práctica, de esta forma queda recluida a las órdenes médicas, negándole prestigio y protagonismo social.

$\checkmark$ La poca utilización del poder por parte de las enfermeras y enfermeros es fundamental para tener una visión global de las implicaciones éticas para la Enfermería como profesión y como sujetos, pues se limita el prestigio profesional y la calidad de los cuidados que la sociedad recibe.

$\checkmark$ Enfermería se ha caracterizado por ejercer labores propias y debe seguir ofreciendo la atención humanitaria que la ha caracterizado, pues el contacto con personas obliga al profesional a ser sensible ante las necesidades de los usuarios(as) sin que ello implique perder su profesionalismo

\section{Bibliografía}

1. Albert, M y Albert S. (2002). Enfermería a examen: ¿Como nos ve la sociedad?: Madrid: http://www.cuidamosweb.com/enfermeriaaexame n.htm.

2. Banton, M. (1971). El rol en la vida social. Buenos Aires: Editorial Troquel

3. Barrantes, R. (2002). Investigación. Un camino al conocimiento. San José: EUNED. 
4. Cano-Caballero G. (2004) Nursing and gender, time for thinking in order to change. Artículo internet vol.13, no.46 [cited 27 May 2006], http://wwwscielo.isciii.es/scielo.

5. Carterette, E y Friedman, M. (1982). Manual de Percepción. Raíces históricas y filosóficas. México D.F.: Editorial Trillas.

6. Colegio Oficial de Enfermeras de Málaga. Desarrollo de la Enfermería en la provincia de Málaga: percepción de los profesionales. Málaga. Recuperado 20 de setiembre de 2005 en http://www.colegioenfermeriamalaga.

7. Chaverri, R y Sánchez, A. (1998). Análisis del Modelo Gerencial desarrollado por los EBAIS en el contexto del Planteamiento de la Reforma del Sector Salud. San José.

8. Enciso Huerta, V. (1997) Género y trabajo: la Enfermería Revista La Ventana, núm. 6 Recuperado Mayo de 2006 http://www.publicaciones.cucsh.udg.mx/

9. Daza R, Torres P, Prieto de R. (2005) Análisis crítico del cuidado de enfermería. Interacción, participación y afecto. Revista Index http://www.index-f.com/index-enfermeria.

10. Cano-Caballero Galvez, María Dolores (2004) Nursing and gender, time for thinking in order to change. Revista Index vol.13, no.46 http://wwwscielo.isciii.es/scielo.

11. Day, R.H. (1983). Psicología de la percepción. México D.F: Editorial Limusa

12. García B, Sains O, Botella R. (2004) La Enfermería vista desde el género. Revista Index vol.13, no. 46

13. González C, Delgado H, y Ayala A. V. (2001) Percepción de la imagen social de la enfermera en la población de Monterrey y su área metropolitana. Reunión Delegacional de Investigación Médica, Monterrey, Nuevo León http://www.uanl.mx/publicaciones/respyn

14. Guevara EB, Mendias EL. (A comparative analysis of the changes in nursing practices related to health sector reform in five countries of the Americas. Rev Panam Salud Pública. $2002 ; 12: 347-53$

15. Hernández $\mathrm{V}$, Rodríguez $\mathrm{B}$, Jiménez $\mathrm{P}$ y Rangel M. (2002) Imagen social de la Enfermería. Revista Cubana Enfermería; 18 (3): Dirección Municipal de Salud. http://bvs.sld.cu/revistas/enf/vol19_1_03

16. J.Yañez, y J. Araya (1999). Perspectivas de la Enfermería profesional Revista Enfermería. Revista $\quad \mathrm{N}^{\circ} \quad 110$. http://www.colegiodeenfermeras.cl/pdf/PERSPR OF.pdf.

17. Moreno M, Betancourt M, Solís T, Barrón R, Gómez H. (2002). Percepción de calidad del cuidado de Enfermería. Facultad de Enfermería de la Universidad Autónoma de Nuevo León: Nuevo León. http://www.uanl.mx/publicaciones/respyn

18. Pizarro, G et al. (2003). Estigma social sobre Enfermería. Revista Cubana de Enfermería V.19 No.1 Ciudad de la Habana Enero.-Abril. http://www.monografias.com/trabajos11/esti/esti.s html.

19. Rojas, L. (2004). Investigación Cuantitativa: Antología. San José: Oficina de Asesores en Evaluación de Programas y Proyectos, S.A. 